\title{
Cerebral Erdheim-Chester disease: report of two cases with progressive cerebellar syndrome with dentate abnormalities on magnetic resonance imaging
}

\author{
Eric Pautas, Patrick Chérin, Sophie Pelletier, Marie Vidailhet, Serge Herson
}

\begin{abstract}
Two patients with Erdheim-Chester disease with progressive cerebellar dysfunction and pyramidal signs are reported on. Cerebral MRI showed bilateral increased signal intensity in peridentatal regions on T2 weighted sequences. Both patients had kidney and bone involvement, established on bone biopsy for one. One patient improved with steroid therapy. This contrasts with previous reports, which describe rare neurological manifestations and the failure of different therapeutic approaches.

(f Neurol Neurosurg Psychiatry 1998;65:597-599)
\end{abstract}

Keywords: Erdheim-Chester disease; cerebellar syndrome; pyramidal signs; magnetic resonance imaging

Erdheim-Chester disease is a rare, nonLangerhans form of systemic histiocytosis of unknown aetiology. ${ }^{1}$ It is associated with tissue infiltration by foamy histiocytes. Intracranial manifestations are rare and the usual localisations are the retro-orbital space and hypothalamic involvement with neurogenic diabetes insipidus. Neurological manifestations are less frequent. ${ }^{2}$ Only a few patients with cerebellar dysfunction have been described. ${ }^{3}$ We report on two patients with Erdheim-Chester disease with slowly progressive cerebellar dysfunction, associated with pyramidal symptoms. The most frequent systemic manifestations are bone lesions. They are characterised by bilateral, symmetric sclerosis of the metaphyseal regions of the long bones. The clinical picture ranges from focal bone lesions to systemic disease with life threatening visceral involvement. $^{12}$

\section{Case reports}

PATIENT 1

A 31 year old man was diagnosed in 1986 as having histiocytosis $\mathrm{X}$ because of pulmonary fibrosis and radiological maxillary lytic lesions. Diagnosis was established by pulmonary biopsies. No treatment was given. Three years later, he developed bone pain with radiological sclerotic lesions in the proximal parts of the tibias and distal parts of the femurs. It was considered to be specific bone involvement in histiocytosis X. Chemotherapy (six regimens of vinblastin and corticosteroids, then two regimens of methotrexate) was administered. In 1991 neurological examination showed cerebellar ataxia and bilateral Babinski's signs. In 1994, after three years of progressive neurological dysfunction, diagnosis of Erdheim-Chester disease was made by analysis of biopsies of the femur bones, showing infiltration with foamy histiocytes lacking Birbeck granules and S-100 protein, and with few lymphocytes. From 1990 to 1994 , blood test results showed chronic inflammation: C reactive protein 92 to 140 $\mathrm{mg} / 1$ (normal<4 mg/l), erythrocyte sedimentation rate 50 to $100 \mathrm{~mm}$, and plasmatic fibrin 7 to $13 \mathrm{~g} / 1$. Serum triglyceride concentrations were normal. Abdominal echography and CT showed "fat kidneys" with perirenal infiltration. There was no abnormality on brain CT but cerebral MRI showed abnormal increased signal intensity on $\mathrm{T} 2$ weighted sequences in the sphenoidal region and in both dentate nuclei.

In 1996, bone lesions and radiological findings were unchanged. Cerebellar dysfunction was slowly progressive. The patient was treated with corticosteroids $(10 \mathrm{mg}$ prednisone/day) for 2 years without success.

PATIENT 2

A 62 year old woman without medical history was admitted in December 1995 for biological inflammatory syndrome and ataxia. Neurological examination showed cerebellar ataxia and dysmetria of four limbs, tetrapyramidal syndrome with brisk deep tendon reflexes of four limbs, and bilateral Babinski's signs. There was a right central scotoma secondary to inflammatory optic neuritis. General examination showed bilateral periorbital xanthoma but no tendon swelling.

Laboratory tests found chronic inflammation with C reactive protein of $50 \mathrm{mg} / \mathrm{l}$ (normal<4 mg/l), erythrocyte sedimentation rate of $99 \mathrm{~mm}$, polyclonal hypergammaglobulinaemia, and hypertriglyceridaemia $(4 \mathrm{mmol} / 1$; normal $<1.65 \mathrm{mmol} / \mathrm{l})$. The serum vitamin $\mathrm{E}$ 


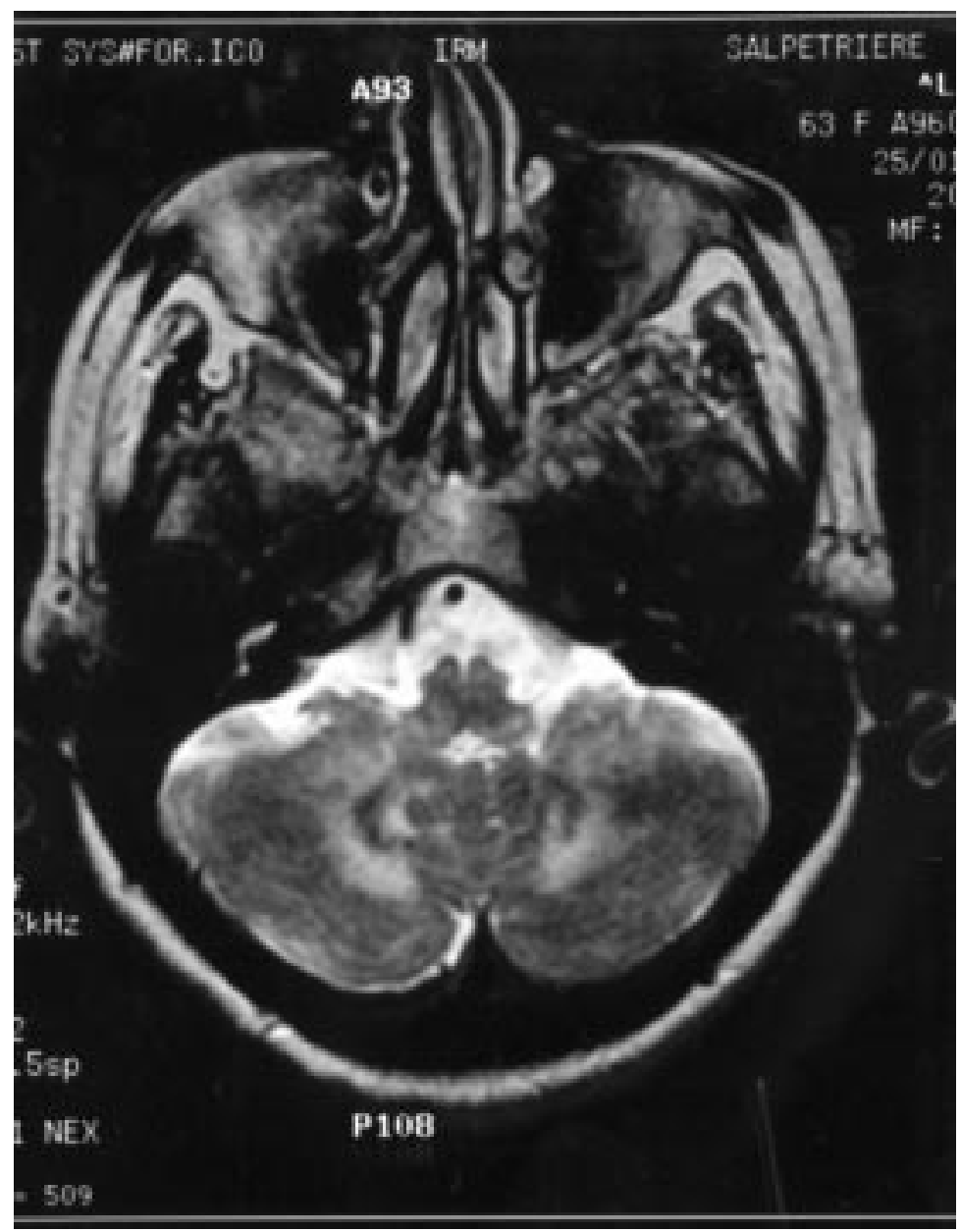

Figure 1 Brain MRI showing bilateral hypersignal of peridentatal regions on $T 2$ weighted sequences, without cerebellar atrophy.

concentration was within normal limits. Thyroid, renal, hepatic, and phosphocalcic functions were normal. Abdominal echography disclosed enlarged renal calices. Brain CT was normal and CSF was normal. The suspected diagnosis was olivopontocerebellar atrophy associated with inflammatory disease. Corticosteroid therapy was initiated in December 1995 ( $1 \mathrm{mg} / \mathrm{kg} /$ day prednisone). Within six months, neurological examination showed a marked regression of cerebellar ataxia and dysmetria but unchanged pyramidal symptoms. Cerebral MRI showed an abnormally increased signal intensity on $\mathrm{T} 2$ weighted sequences in peridentate regions (fig 1) and on the right optic nerve. Scintigraphy with gallium showed an increased uptake in the periorbital, maxillary, and right humeral areas. Skeletal scintigraphy showed symmetrically increased uptake in the distal parts of the femurs, the proximal and distal parts of the tibias, and the face. Bone radiography showed the typical lesions of Erdheim-Chester disease with patchy sclerosis and osteolysis in the distal parts of the femurs and tibias (fig 2). Abdominal CT showed infiltration of kidneys and perirenal tissues without evidence of retroperitoneal fibrosis or obstructions in the ureters.

The patient died a few weeks later with an unexplained metabolic acidosis. The patient

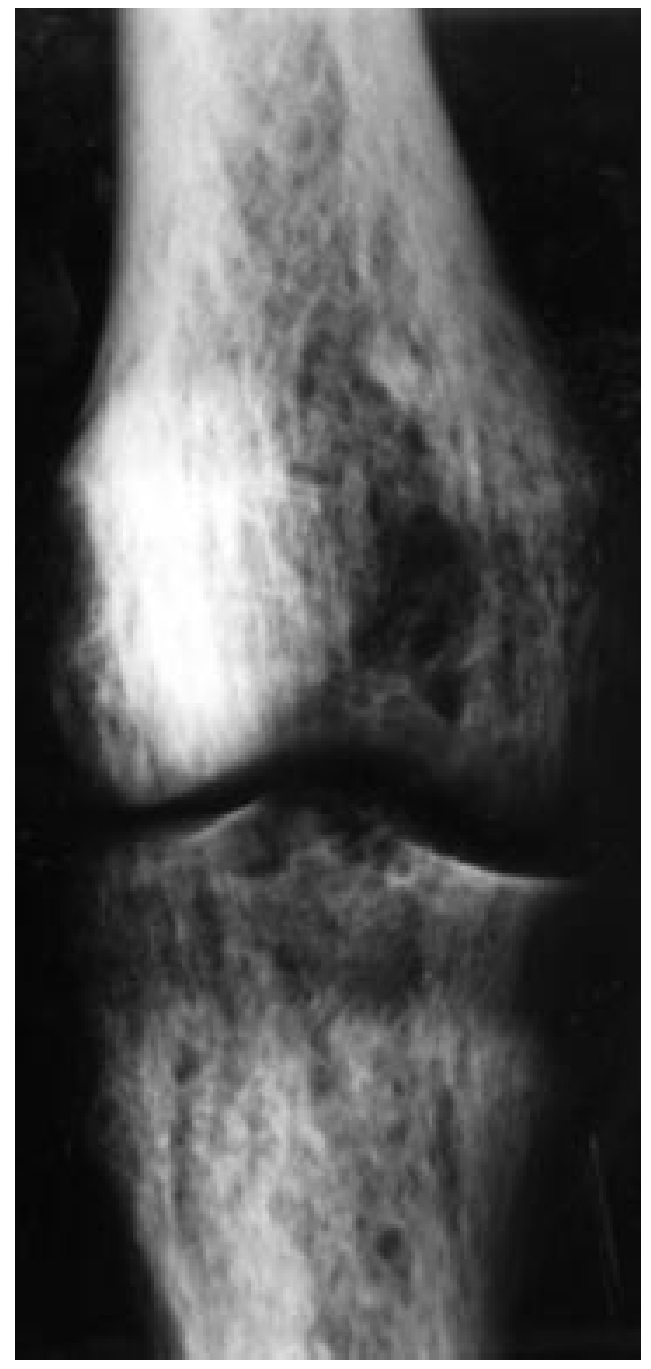

Figure 2 Femur and tibia radiography showing sclerosis of the metaphyseal regions with areas of osteolysis.

was diagnosed as having Erdheim-Chester disease with multisystemic involvement (bone, kidney, CNS).

\section{Discussion}

We report here on two patients with ErdheimChester disease with neurological involvement. Our patients had similar neurological presentations with cerebellar dysfunction associated with pyramidal signs. Cerebral MRI showed bilateral increased signal intensity in the dentate region on $\mathrm{T} 2$ weighted sequences. Both patients had kidney and bone involvement, patient 1 with pathologically established diagnosis of Erdheim-Chester disease on bone biopsy. The clinical and radiological findings for patient 2 are sufficiently characteristic to be diagnostic of Erdheim-Chester disease. ${ }^{2}$

A patient with cerebellar dysfunction has recently been reported by Fukazawa $e^{2} \mathrm{al}^{4}$; a 59 year old woman developed slowly progressive cerebellar dysfunction (with a follow up of 11 years) associated with bilateral Babinski's signs. T2 weighted cerebral MRI showed hyperintensity in both dentate nuclei. This type of neurological involvement of Erdheim-Chester disease has been reported a few times before. ${ }^{3}$ 
Other neurological manifestations are rare. Three patients had motor deficits, hemiparesis, or paraplegia ${ }^{5}$; two patients were confused ${ }^{7}$; four patients had ataxia or impaired coordination, but the authors did not state whether they had cerebellar or proprioceptive dysfunction. ${ }^{8-10}$

Neuroradiological findings indicate brain infiltration in four patients. Brain MRI findings include isosignal on $\mathrm{T} 1$ weighted sequences with enhancement after gadolinium, and increased signal intensity on $\mathrm{T} 2$ weighted sequences. ${ }^{68}$ The other localisations for intracranial accumulation of histiocytes were the retro-orbital space with exolphtalmos (16 patients) or diplopia (four patients), and the hypophyso-hypothalamic area with diabetes insipidus (17 patients). ${ }^{2}$

The most frequent systemic histiocytosis is histiocytosis $\mathrm{X}$, now called Langerhans cell histiocytosis, which includes three entities: Hand-Shüller-Christian disease, Letterer-Siwe disease, and eosinophilic granuloma. ErdheimChester disease differs from Langerhans cell histiocytosis by the immunohistological and microscopical characteristics of the histiocytes. Erdheim-Chester disease is a rare multisystemic xanthogranulomatous infiltrative disease of unknown aetiology, due to infiltration of different organs and bones by foamy histiocytes. ${ }^{1}$ The extraskeletal involvement is various: enlarged kidneys by renal infiltration, hydronephrosis, retroperitoneal fibrosis, pelvic infiltration, pulmonary interstitial infiltration or fibrosis, pleural thickening or effusion, pericardial effusion, intracranial infiltration. ${ }^{2}$

A total of 59 patients have been reported. In a review of the literature, Veyssier- Belot et al showed that only 11 of these patients fulfilled all the diagnostic criteria for Erdheim-Chester disease. ${ }^{2}$ The specific signs are radiological and histological features in bone. The characteristic radiographical finding is bilateral, symmetric osteosclerosis of the long bones. Osteolytic lesions are described in Langerhans cell histio- cytosis but not in the long bones. Full histological criteria are xanthomatous or xanthogranulomatous infiltration by fibrosis with lack of S-100 protein and of intracytoplasmic Birbeck granules, both described in Langherans cell histiocytosis. ${ }^{12}$

The pathogenesis of neurological manifestations in Erdheim-Chester disease remains an unknown and intriguing issue particularly in the light of the improvement of cerebellar symptoms after corticosteroid therapy for 2 months, which has not been previously reported. Our findings broaden the clinical range of known neurological manifestations in Erdheim-Chester disease and suggest that hyperintensity of dentate nuclei on MRI in association with pseudoprogressive olivopontocerebellar degeneration could suggest the diagnosis of Erdheim-Chester disease. Skeletal survey of the long bones and ophtalmological examination are indicated for such patients to confirm the diagnosis.

1 Jaffe HL. Gaucher's disease and certain other inborn metabolic disorders : Lipid (cholesterol) granulomatosis. In : Jaffe HL, ed. Metabolic, degenerative, and inflammatory diseases of bones and joints. Philadelphia: Lea and Febiger, 1972:535.

2 Veyssier-Belot C, Cacoub P, Godeau P, et al. ErdheimChester disease. Clinical and radiologic characteristics of 59 cases. Medicine 1996;75:157-69.

3 Smith ME, Katz DA, Harris JO, et al. Systemic histiocytosis presenting as multiple sclerosis. Ann Neurol 1993;33:54954.

4 Fukazawa T, Tsukishima E, Sasaki H, et al. ErdheimChester disease and slowly progressive cerebellar dysfunction. F Neurol Neurosurg Psychiatry 1995;58:238-40.

5 Elian M, Bornstein B, Matz S, et al. Neurological manifestations of general xanthomatosis. Arch Neurol 1969;21:115-

6 Caparros-Lefebvre D, Pruvo JP, Remy M, et al. Neuroradiologic aspects of Erdheim-Chester disease. Am $\mathcal{F}$ Neuroradiol 1995;16:735-40.

7 Poehling GG, Adair DM, Haupt HA. Erdheim-Chester disease. A case report. Clin Orthop 1984;185:241-44. 8 Tien RD, Brash RC, Jackson DE, et al. Cerebral Erdheim-Chester disease: persistent enhance

9 Lantz B, Lange TA, Heiner J, et al. Erdheim-Chester disease: a report of three cases. $\mathcal{F}$ Bone foint Surg 1989;71: 456-64

10 Masshof W. Das gehirn bei der lipoidgranulomatose. Beitr Path Anat 1948;110:544-65. 